\title{
Session 21.2 - Measurement of Light at Night
}

\author{
Richard J. Wainscoat \\ University of Hawaii, Institute for Astronomy \\ email: rjw@IfA.Hawaii.Edu
}

\begin{abstract}
The introduction of the mercury vapor lamp for general lighting in the 1930s probably marked the beginning of significant light pollution. Lighting levels have increased slowly, year-toyear, with sky brightness levels increasing only slowly on timescales of a year; no measurement protocols or instruments existed to quantify this increase. However, on timescales of 10-20 years, or on generational timescales, the increases in night sky levels, particularly in urban areas, have been dramatic. Younger people speak with their parents or grandparents who remark how beautiful the sky used to be, and how many stars they could see when they when they were younger. Older people can themselves remember how many stars were visible in the sky when they were younger. Whole generations of children now grow up without ever seeing the Milky Way.

Society has not had tools to easily measure sky brightness, and monitoring from space has only recently become available. A subtle increase of $10 \%$ sky brightness per year, for example, is not noticeable to the human eye on the time scale of a year, and has been tolerated by society. But such an increase compounds to an increase of a factor 2.6 in 10 years, 6.7 in 20 years, and a factor 45 in 40 years, corresponding to a dramatic increase in sky brightness, an almost complete loss in ability to see faint objects in the night sky, and rendering the sky unusable for most forms of astronomy. The most striking examples are the urban observatories found in many major cities that can no longer be used.

Session 2 was primarily focused on measurement of light at night, with an emphasis on measurement of light pollution. It comprised of 6 papers that are summarized below. Over the last decade, our ability to measure light pollution has grown tremendously, and the instrumentation needed to produce reliable quantitative measurements has become much more affordable, and now includes consumer grade digital cameras and even smart phones. During this same time period, light pollution has continued to grow. The widespread changes from mostly highpressure sodium lighting to LED lighting that are now occurring make continued monitoring and measurement of light pollution particularly important into the future.

Complete presentations may be viewed at: http://www.noao.edu/education/IAUGA2015FM21
\end{abstract}

Keywords. light pollution, measurement

\section{Derivation of sky quality indicators from photometrically calibrated all-sky image mosaics}

Authors: D.M. Duriscoe, C.A. Moore, Night Skies Program, U.S. National Park Service, Bishop, California, United States; C.B. Luginbuhl, Dark Sky Partners, L.L.C., Tucson, Arizona, United States

For many years, the US National Park Service Night Skies Program has been acquiring carefully calibrated mosaic images of the night sky. A large database of high resolution all-sky measurements of $V$-band night sky brightness at sites in U.S. National Parks and astronomical observatories, including a few international locations, has been amassed. This database can be utilized to describe sky quality over a wide geographic area. 


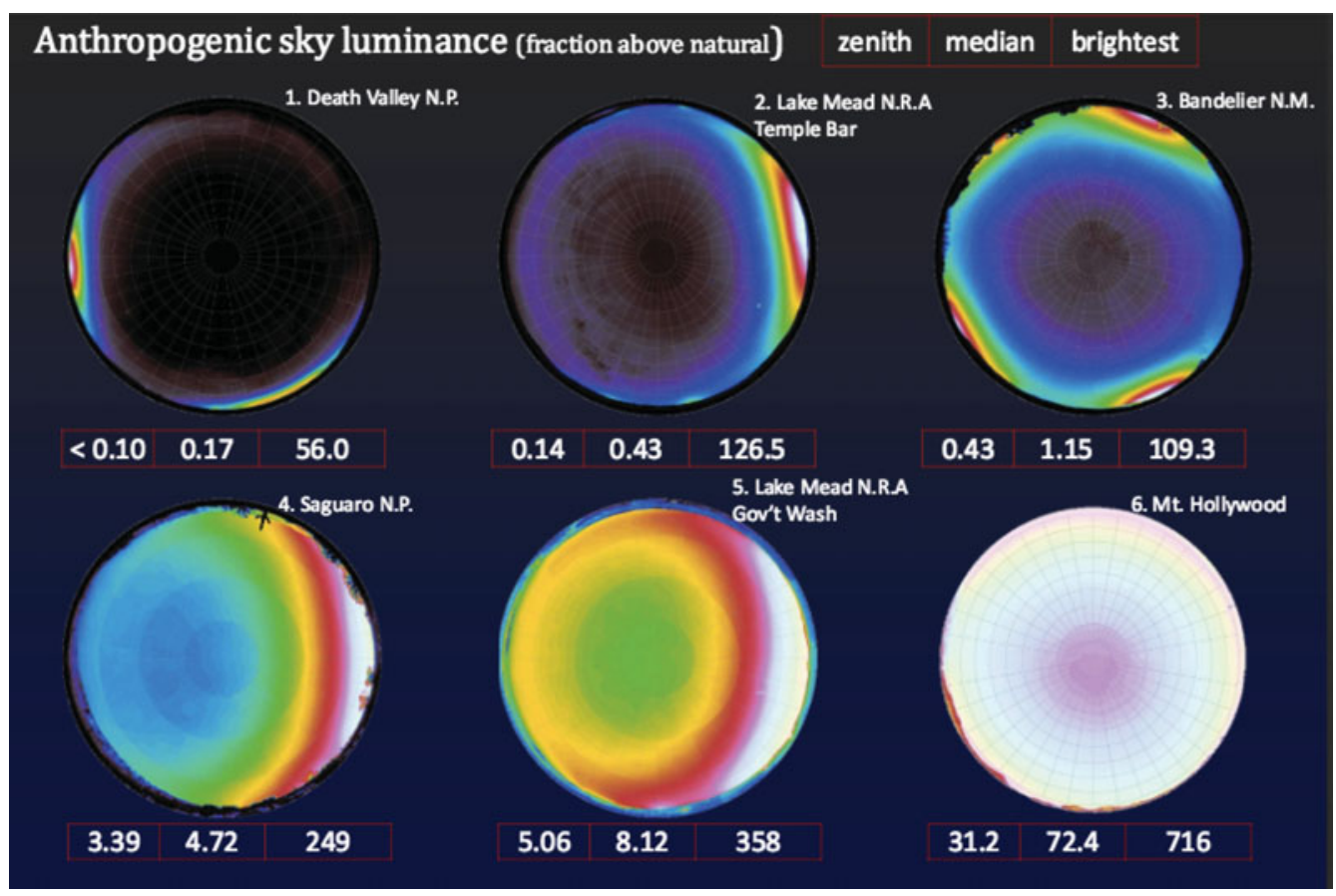

Figure 1: Artificial Sky Luminance

Mosaics of photometrically calibrated $V$-band imagery are processed with a semiautomated procedure to reveal the effects of artificial sky glow through graphical presentation and numeric indicators of artificial sky brightness (Figure 1). Comparison with simpler methods such as the use of the Unihedron SQM and naked eye limiting magnitude reveal that areas near the horizon, which are not typically captured with single-channel measurements, contribute significantly to the indicators maximum vertical illuminance, maximum sky luminance, and average all-sky luminance.

Artificial skyglow at the zenith only is difficult to measure accurately because of variations in the natural airglow, and because artificial skyglow is typically lowest at the zenith.

Distant sources of skyglow may represent future threats to areas of the sky nearer the zenith. The brightest area of the sky is a very sensitive indicator of visual intrusion and points to areas of the sky for continuous monitoring where trends can be easily detected. Timely identification and quantification of these threats may allow mitigating strategies to be implemented.

Astronomers should establish a median all sky limiting value for light pollution at research-grade observatories. A value of no more than $20 \%$ above natural is suggested. A smaller value, such as no more than $10 \%$ above natural is highly desirable for the best astronomical observatory sites where 4-meter or larger telescopes are located.

Expansion of this work beyond the $V$ filter will be needed in the future to properly quantify the effects of changes of lighting to Light Emitting Diodes (LEDs). White LEDs emit blue light that is particularly damaging both to astronomy and to the dark adapted scotopic human eye's view of the night sky, and this blue emission, centered at $450 \mathrm{~nm}$, falls outside the transmission curve of the $V$ filter. 


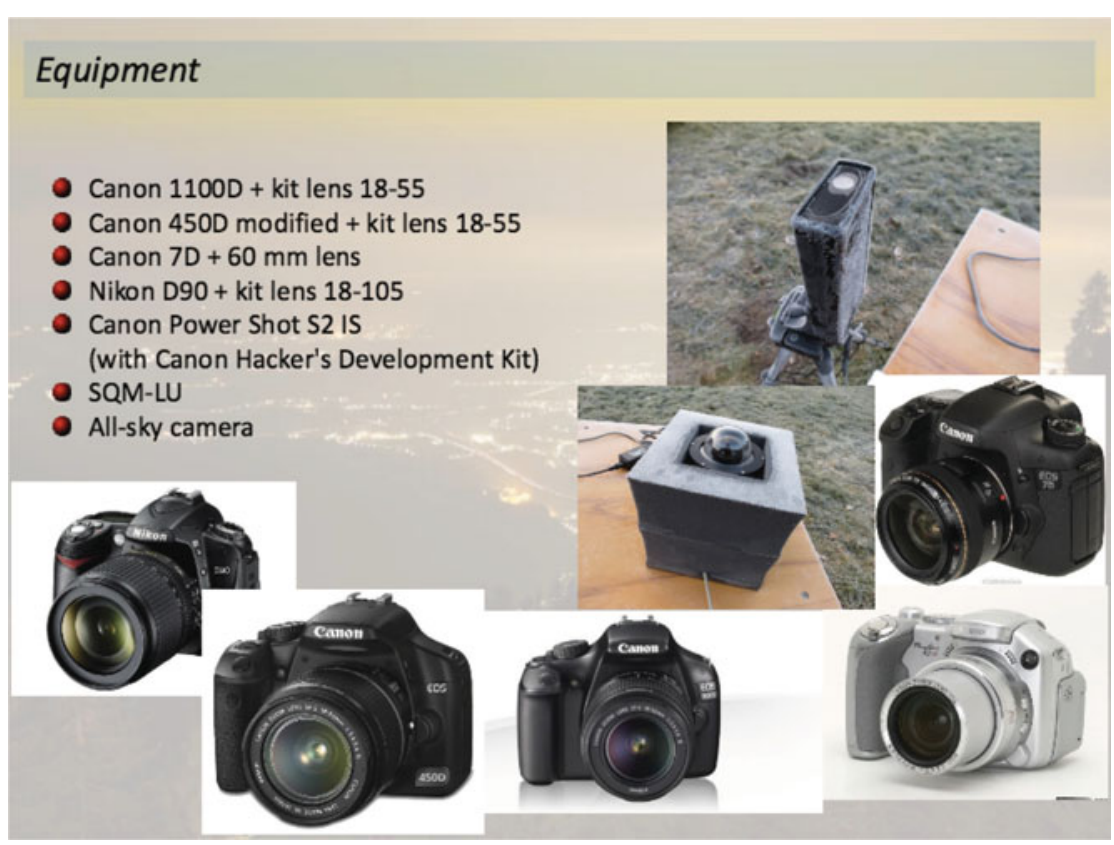

Figure 2: DSLR cameras used in measuring night sky brightness.

\section{Night sky photometry with amateur-grade digital cameras}

Authors: T. Mrozek, M. Steslicki, Solar Physics Division, Space Research Centre PAS, Wroclaw, Poland, T. Mrozek, D. Gronkiewicz, S. Kolomanski, Astronomical Institute, University of Wroclaw, Wroclaw, Poland

Measurements of night sky brightness can give us valuable information on light pollution. The more measurements we have, the better is our knowledge on the spatial distribution of the pollution on local and global scales.

High accuracy professional photometry of night sky can be performed with dedicated instruments. The main drawbacks of this method are high price and low mobility. The apparatus used by the National Park Service in Section 1 above, for example, consists of an astronomy grade cooled CCD, heavy mount, computer and portable power supply. It is expensive and not easily portable, and beyond the reach of most people.

This limits the amount of observers and observations and therefore the amount of photometric data that can be collected. In order to overcome the problem of the limited amount of data, we can involve amateur astronomers in photometry of the night sky. However, to achieve this goal we need a method that utilizes equipment that is usually used by amateur astronomers - digital cameras.

Digital cameras have improved tremendously over the last decade, with both professional and amateur grade digital cameras now having very low noise performance. The noise performance of these digital cameras is temperature dependent, and their noise is lower at colder temperatures (albeit with poorer battery performance at these colder temperatures).

We propose a method that enables good accuracy photometry of night sky with a use of digital compact or digital single lens reflex (DSLR) cameras. (See Figure 2.) Reduction of observations and standardization to $B V R$ system are performed.

We tested several cameras, including popular compact digital cameras and amateur 
grade DSLRs in the Izera Dark Sky Park in Poland. We compared our results to Sky Quality Meter (SQM) measurements. The overall consistency for results is within 0.2 mag. Our method is very simple but is quantitative and uses inexpensive equipment that is already owned by many people including amateur astronomers.

We tested our methodology with secondary school students, a group of teachers, and a group of amateur astronomers. This allowed us to simplify the method and to identify the most problematic issues that could lead to degradation of results. We believe that this method can be deployed in a widespread manner to obtain a substantial number of light pollution measurements over wide areas and spanning long periods of time to show long terms trends in the sky brightness.

\section{Evaluation of the night sky quality at El Leoncito and LEO++ in Argentina}

Authors: M. Aubé, N. Fortin, S. Turcotte, Physics, Cégep de Sherbrooke, Sherbrooke, Quebec, Canada, B. Garcia, A. Mancilla, J. Maya, CNEA-CONICET-UNSAM, Instituto de Tecnologas en Deteccion y Astropartculas, Mendoza, Argentina

Light pollution is a growing concern at many levels, but especially for the astronomical community. Artificial lighting veils celestial objects and disturbs the measurement of night time atmospheric phenomena. This was the motivation for this sky brightness measurement experiment in Argentina. The goal was to determine the quality of two Argentinian observation sites: LEO++ and El Leoncito. (See Figure 3.) Both sites were candidates to host the Cherenkov Telescope Array (CTA). This project consists of an arrangement of many telescopes that can measure high-energy gamma ray emissions via their Cherenkov radiation produced when entering Earth's atmosphere. A very dark sky is essential for this experiment.

Even if the two Argentinian sites had been excluded from the final CTA site competition, they are still of great interest for other astronomical projects. Especially the El Leoncito site which already hosts the Leoncito Astronomical Complex (CASLEO). Both sites are in western Argentina, close to the Chilean border. The closest large town is San Juan.

The measurement methods used to determine the sky quality were described in detail. These included two different techniques: Sky Quality Meters (SQM) and the Spectrometer for Aerosol Night Detection (SAND). Seasonal variations in sky brightnesses at El Leoncito were clearly observed, with the fall season having an average sky brightness 0.3 magnitudes brighter than average. The darkest skies were in the summer. The variation in sky brightness is related to seasonal variations in aerosol content in the atmosphere.

The results were compared to different renowned astronomical sites (Kitt Peak, Arizona, USA, and Mont-Mégantic, Canada). The two Argentina sites studied have similar sky brightness levels to Kitt Peak and Mont Mégantic. We found that $\mathrm{LEO}++$ is a high quality site, however there are a lot of aerosols that can interfere with astronomical measurements. El Leoncito shows very low sky brightness levels, which are optimal for low light level detection, and has the highest potential for future development as an astronomical site. It shows low radiance in the $569 \mathrm{~nm}$ sodium line, low aerosol content (resulting in lower extinction) and is surrounded by mountains that suppress the impact of the surrounding luminous halos. 


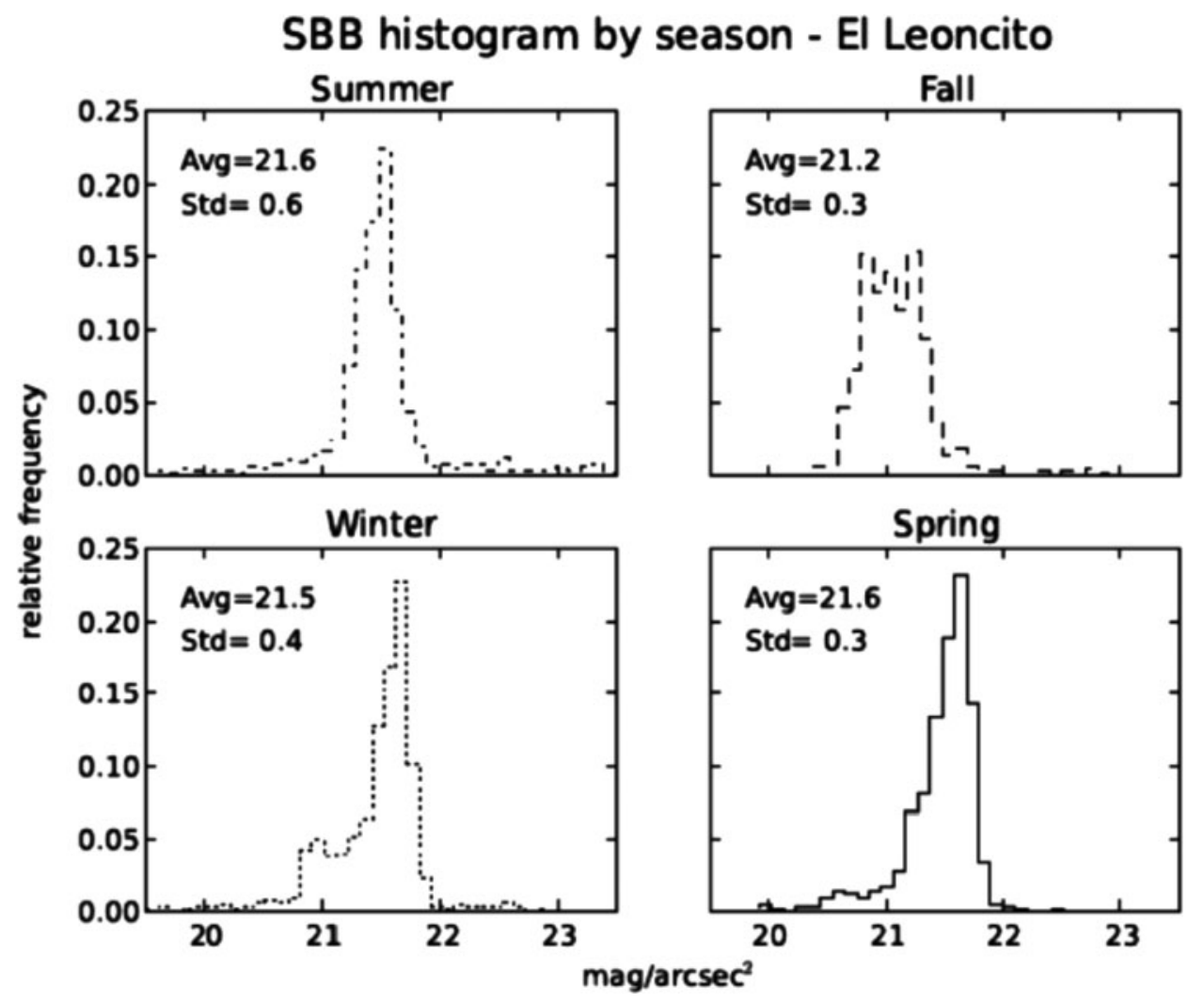

Figure 3: Seasonal behavior of the night sky brightness at El Leoncito.

\section{Globe at Night - Sky Brightness Monitoring Network}

Authors: S. Cheung, IAU Office for Astronomy Outreach, Mitaka, Tokyo, Japan, S. Cheung, Y. Shibata, H. Agata, National Astronomical Observatory of Japan, Tokyo, Japan, J.C. Pun, C. So, The University of Hong Kong, Hong Kong, Hong Kong, C.E. Walker, National Optical Astronomy Observatory, Tucson, Arizona, United States

The Globe at Night - Sky Brightness Monitoring Network (GaN-MN) is an international project for long-term monitoring of night sky conditions around the world. The GaN-MN consists of fixed monitoring stations each equipped with a Sky Quality Meter - Lensed Ethernet (SQM-LE), which is a specialized light sensor for night sky brightness (NSB) measurement. NSB data are continuously collected at high sampling frequency throughout the night, and these data are instantly made available to the general public to provide a real-time snapshot of the global light pollution condition. A single data collection methodology, including data sampling frequency, data selection criteria, device design and calibration, and schemes for data quality control, was adopted to ensure uniformity in the data collected. This is essential for a systematic and global study of the level of light pollution. The data collected also provide the scientific backbone in our efforts to contribute to dark sky conservation through education to the general public and policy makers. The GaN-MN project is endorsed by the IAU IYL Executive Committee Working Group as a major Cosmic Light program in the International Year of Light.

The data are collected using the Unihedron lens-ethernet sky quality meters. These provide sky brightnesses in units of mag $\operatorname{arcsec}^{-2}$, with an accuracy of \pm 0.1 mag $\operatorname{arcsec}^{-2}$. 


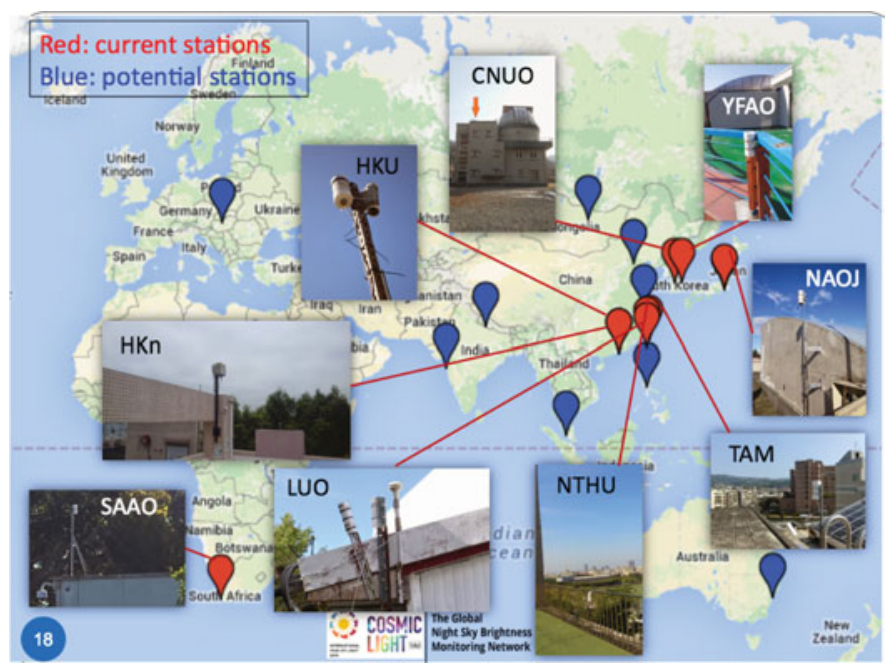

Figure 4: Current and potential sky brightness monitoring station locations worldwide

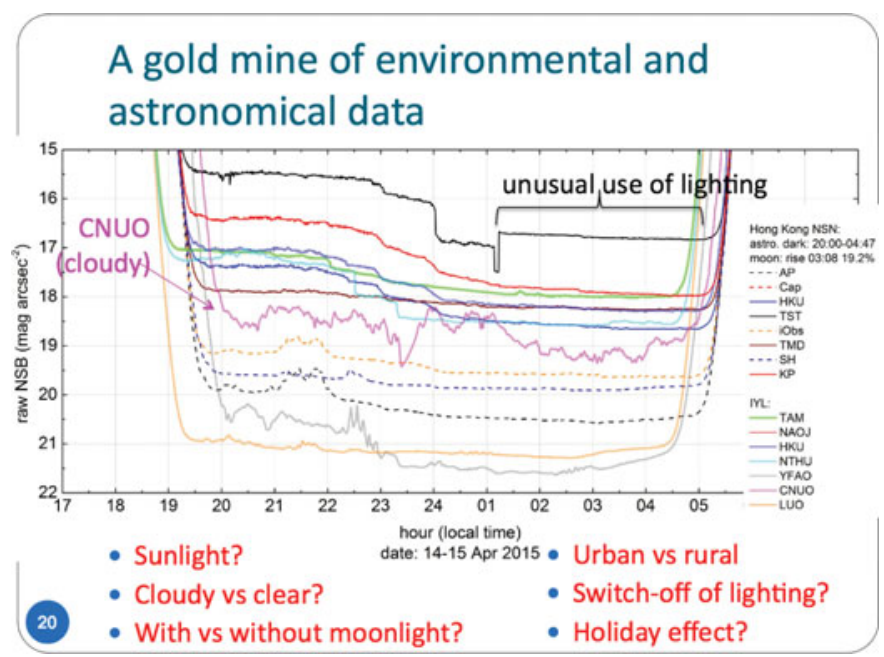

Figure 5: The Sky Quality Meter measurements provide a plethora of environmental and astronomical data.

The SQM-LE has a spectral response function centered at $540 \mathrm{~nm}$, with a full-width halfmaximum of $240 \mathrm{~nm}$, roughly corresponding to the spectral sensitivity of human photopic vision. On the sky, the SQM-LE samples a circular area with full-width half-maximum of $20^{\circ}$.

The SQM-LE was chosen for its modest cost $(\$ 250)$ and robustness. A weatherproof housing provided by the manufacurer allows standardized installation and data reduction. A short (30-second) sampling interval has been adopted to record short-term sky brightness variations. Date from the SQM-LE is sent directly to a cloud-based database. Infrastructure requirements are minimal (power, internet and mount); maintenance is minimal - regular calibration is needed to maintain data quality.

Data are available in real-time via a public interface, and have been integrated into the Google Map platform. Existing stations include locations in Taiwan, Japan, Hong Kong, 
South Korea and South Africa. Many other potential sites exist, and new installations are strongly encouraged with the aim of producing a live light pollution map of the world. (See Figures 4 and 5.)

\section{Dark Sky Meter - International Year of Light 2015 app}

Authors: P. Russo, T. Sankatsing Nava, Leiden Observatory, Leiden University, Leiden, Netherlands

Many people now have smart phones, and the intent of the Dark Sky Meter app is to provide everyone who has a smart phone an easy way to measure the brightness of the night sky. The cameras in smart phones have improved dramatically in their capabilities over the last few models of phone, including better optics and lower noise levels.

The Dark Sky Meter IYL 2015 edition app measures the night sky brightness using a smart phone. The app is presently limited to the Apple iPhone. The principal reason for not expanding the app to the larger Android phone market is related to hardware. The iPhone has a single manufacturer (Apple), whereas Android phones are made by many different companies. As a result, there are only a few variants of the camera in iPhones (corresponding to different models of iPhone), but there is a very diverse range of cameras in Android phones. This made it impossible, within the scope of the current app, to expand usage to the cameras in Android phones. This, of course, limits the app to only iPhone owners. However, the iPhone presently has a market share of approximately $40 \%$, so the app can be used by many people.

The night sky brightness measured by the Dark Sky Meter app was compared to measurements from the Unihedron Sky Quality Meter (SQM) over a range of sky brightness spanning 15.5-21.5 mag $\operatorname{arcsec}^{-2}$. Some systematic differences are evident, but these can be corrected in future versions of the app. (See Figure 6.)

From the reviews of the app, it is clear that some users are trying to use the app under street lamps or under a full moon. This produces inconsistent and unusable results, and also produces user frustration, producing the illusion that the app is not useful and flawed.

Plans for the future (version 2.0) include user interface improvement and better instructions, analysis of the data, finding funding the keep the app free, developing educational material, and demonstrating the relationship between sky brightness and energy waste. An observing campaign is planned for September 2015 during the International Year of Light 100 hours of light.

\section{6. in situ Measures of LED Installations: Results of Air and Ground Surveys}

Authors: E.R. Craine, B.L. Craine, Western Research Company, Tucson, Arizona, United States, E.R. Craine, B.L. Craine, STEM Laboratory, Inc., Tucson, Arizona, United States

Light Emitting Diode (LED) outdoor light fixtures of different types are rapidly proliferating in many communities, particularly in the form of continuous roadway, work, and parking lot lights. These lights offer a wide range of benefits, but many in the astronomical community have expressed various concerns about their impact on local observatory facilities. We have spent several years developing complementary ground-based and aerial techniques of measuring light installations in the field. Unfortunately, large community retrofits of lighting preclude comprehensive measurement of the changes that result unless 
Performance: SQM vs DSM
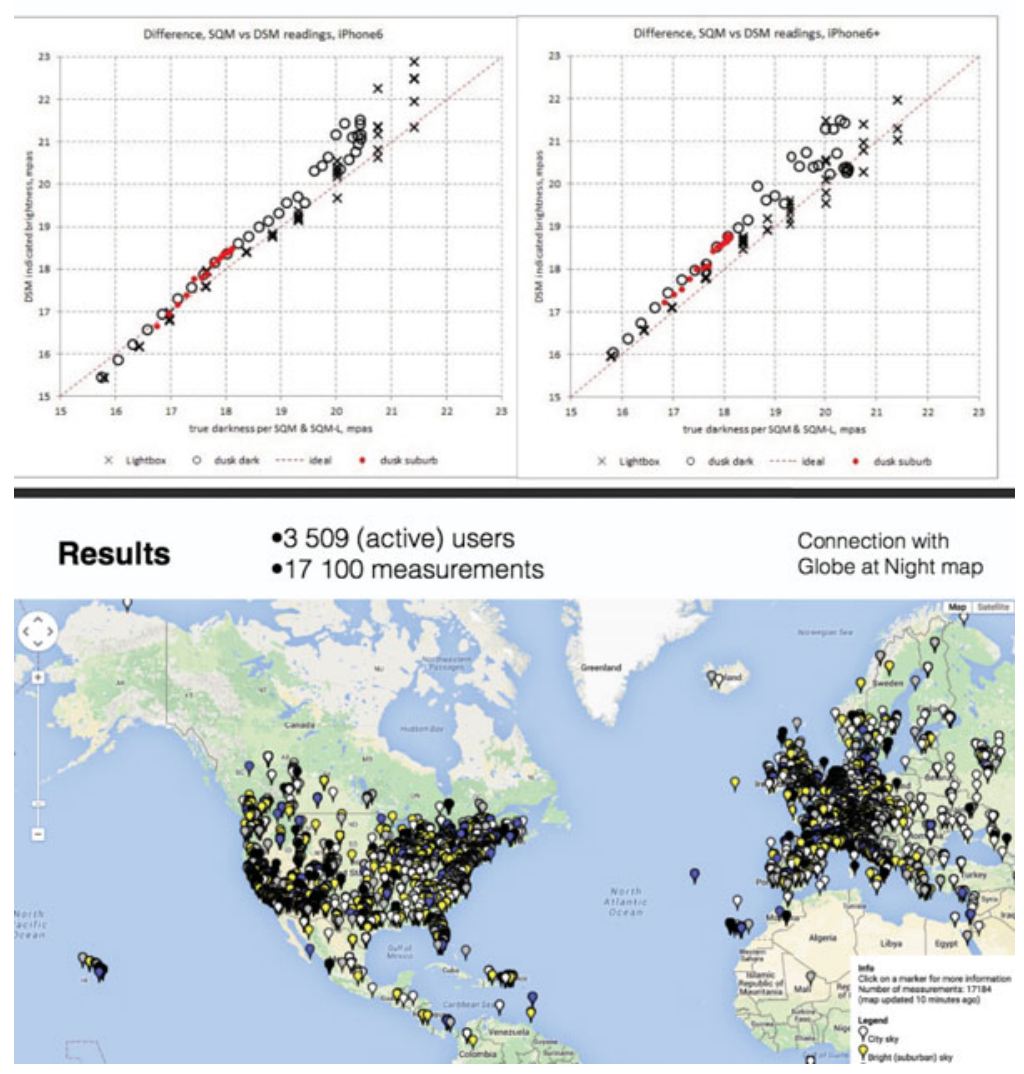

Figure 6: The Dark Sky Meter app performance compared with Sky Quality Meters' plus a world map of over 17,000 measurements

baseline data have been collected prior to completion of the new installations. Because of the rapidity of conversion to LEDs, it is increasingly difficult to conduct informative before and after surveys.

Our LED measurement projects include the Rosemount Copper Mine, Tucson International Airport, and Sierra Vista (all in Arizona, US), and Waikoloa Village and Kawaihae Harbor (both in Hawaii, US). The measurement protocols include static ground surveys, mobile ground surveys, airborne surveys, and satellite data surveys. The Hawaii locations are undergoing changes from low-pressure sodium lighting to filtered LED lighting.

The survey data show, for example, that LED lighting is typically much more uniform than high-intensity discharge lighting (e.g., high-pressure sodium), and can also highlight installation or design errors.

As a point of interest to astronomers, we offer examples of some in situ measurements of LED installations, compare those measurements to results for older light fixtures, and discuss some of the implications for astronomy. These objective data may be helpful in reaching an informed perspective on how LED lights perform in typical settings.

Quantitative in situ measurements are pivotal to understanding the impact of lighting strategies on artificial light at night. Although models are interesting, real observational data lead to the truth. LEDs are the lighting of the future because of their economics. But the spectral energy distribution of LEDs is less desirable from the dark sky perspective. 


\section{Zenith Angle Function}

\section{TIA LED Retrofit Area}

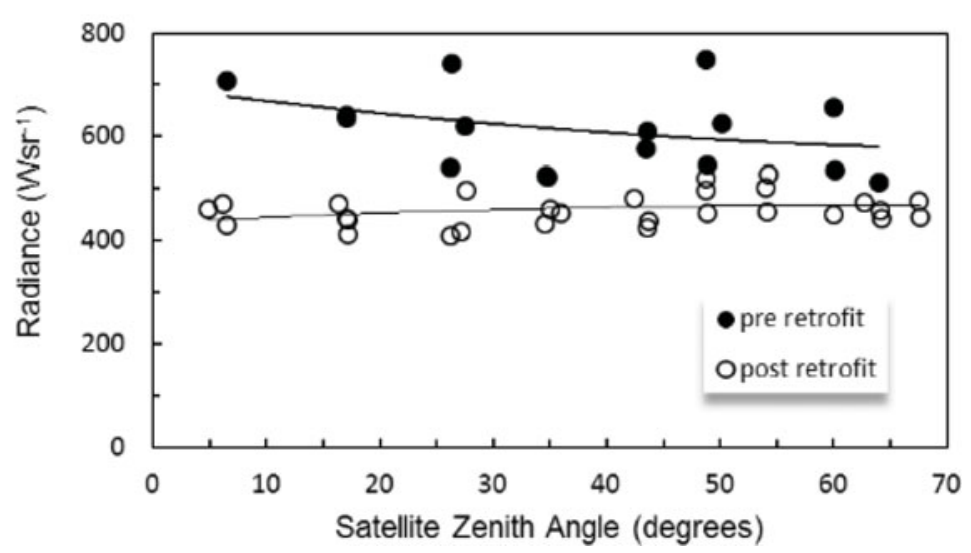

Figure 7: The satellite zenith angle function versus radiance for the Tucson International Airport (TIA) "LED Retrofit Area".

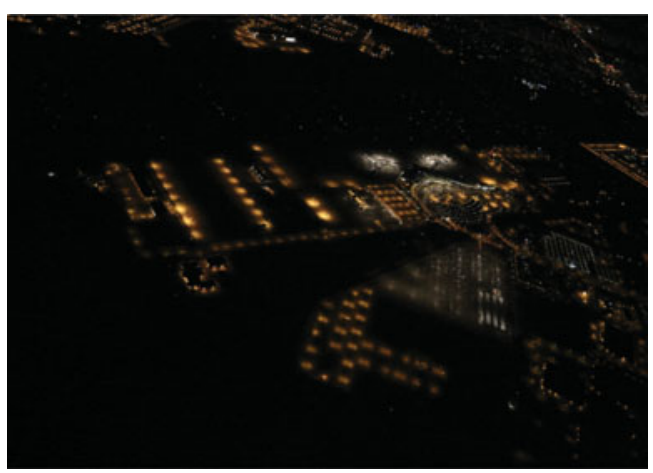

Figure 8: Seeing the task oriented footprints on the ground of the parking lot area of TIA from the Airborne Survey.

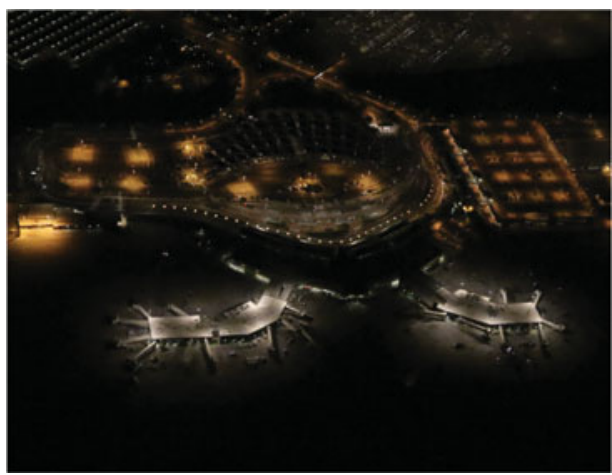

Figure 9: Seeing the task oriented footprints on the ground of the terminalarea of TIA from the Airborne Survey.

LEDs can reduce overall brightness levels. A full cutoff design is not the same as a full cutoff installation. Multimodal quantitative measurement of installed fixture removes the ambiguity about installations, provides feedback for improvement, and allows monitoring to validate the status of light installations. 